\title{
EXPERIMENTAL RESEARCH OF A MICROJET CAVITATION
}

\author{
Branislav KNÍŽAT, Róbert OLŠIAK, Marek MLKVIK•
}

\begin{abstract}
The paper presents some results of a cavitation research behind a micro-orifice. Investigated were the conditions of the origin of cavitation represented by parameters such as upstream pressure, downstream pressure, liquid temperature and cavitation number. Presented are also images of a cavitating microjet made by the high speed high definition camera RedLake Y3. Dimensions of a microjet are: diameter 0,3 $\mathrm{mm}$; length $0,5 \mathrm{~mm}$.
\end{abstract}

\section{INTRODUCTION}

Recently a great attention is paid to a research of the cavitation in microdevices. It is related to a development of microfluidic applications and also with an exploration of the cavitation as a useful phenomenon: surfaces cleaning, material disintegration, cold disinfection, etc. We dealt in our institute with the research of cavitation in micro-orifices for purpose of development of a device intended to a munition disposal and rock drilling. Results of the research are applicable in a jet optimization for purpose of maximization of required effect of cavitation. This paper presents a test rig where the research was done and some results as well.

\section{RESEARCH METHODOLOGY}

The research consisted of three main parts: CFD simulation, flow visualization, and study of other effects such as acoustic (ultrasound) emissions. This paper dealt with the experimental research of cavitation behind a micro-orifice at very low cavitation numbers when a cavitation cloud is drifted from the space of the orifice and collapses at some distance behind it (supercavitation). Respect to velocities in a micro-orifice (up to 100 $\mathrm{m} / \mathrm{s}$ ) and respect to its dimensions the flow was photographed by a high speed high definition camera. Erratic length of the cavitation cloud was analysed from the camera images. Acoustic effects were recorded by an ultrasound sensor of acoustic emissions. As a liquid was used water with known temperature.

\section{TEST RIG DESCRIPTION}

In the Fig. 1 is the scheme of the test rig. A base part is a high pressure pump supplying water to the microfluidic chamber where is placed the micro-orifice. Water then flows to the drain. The space behind the orifice is photographed by a high speed HD camera RedLake Y3. Pulsed light source is placed under the transparent chamber. The camera is connected to the computer where the images are saved. Following parameters were also measured:

\footnotetext{
- Branislav Knížat (branislav.knizat@stuba.sk), Róbert Olšiak (robert.olsiak@stuba.sk), Marek Mlkvik (marek.mlkvik@stuba.sk)
} 
Input pressure P1 - piezoresistive pressure sensor (BD Sensors), Range: 0-600 bar, Output: 0-10 V; Output pressure P2 (in chamber) - piezoresistive pressure sensor (BD Sensors), Range: 0-1 bar, Output: 0-10 V. Mass flow rate: Weighing machine UWE GM$11 \mathrm{~K}$, sensitivity $1 \mathrm{~g}$.

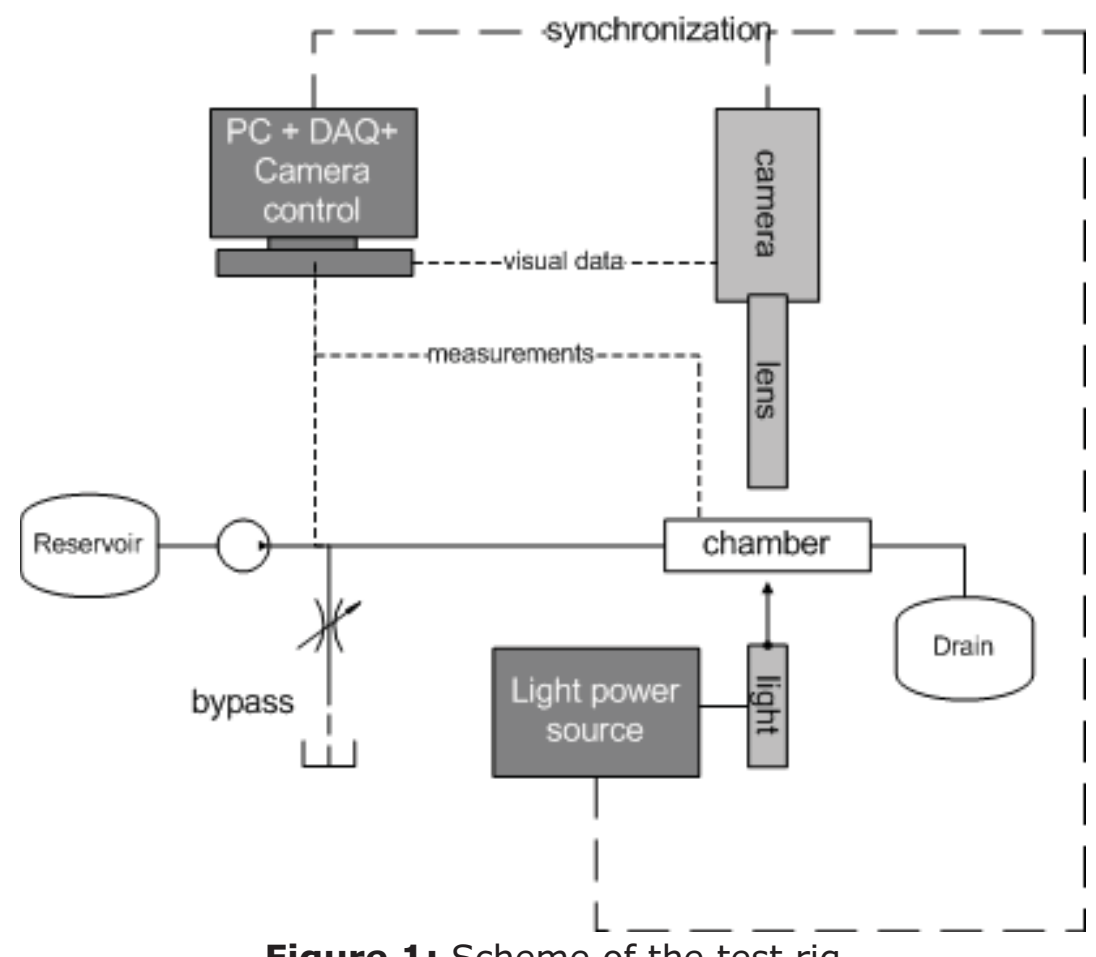

Figure 1: Scheme of the test rig
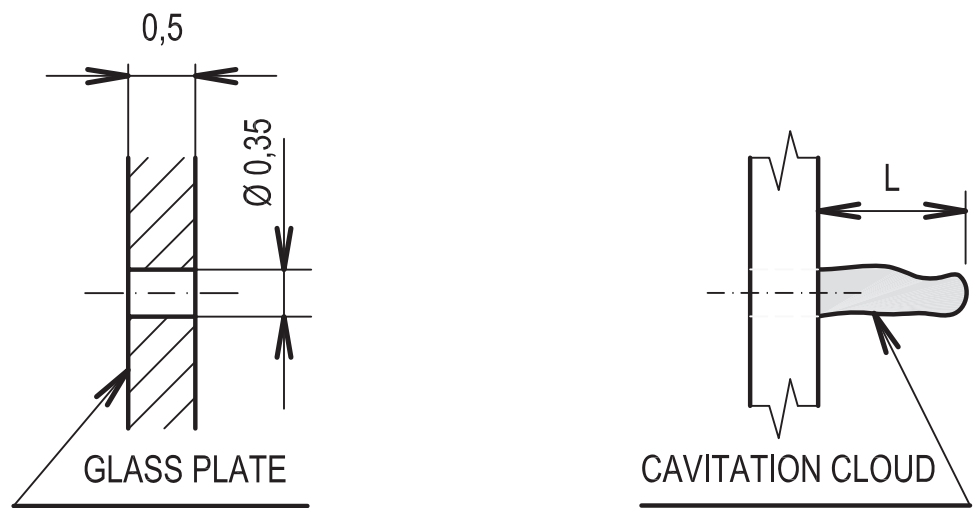

Figure 2: Tested micro-orifice (left). Cavitation cloud behind the micro-orifice (right)

In the fig. 2 left is pictured the micro-orifice with its dimensions. Cylindrical micro-orifice is made in $0,5 \mathrm{~mm}$ thick silicon glass plate. In the figure 2 right is a typical flow pattern at extreme low values of the cavitation number, when bubble cloud is drifted behind the orifice. The temperature was measured during the experiments as a parameter necessary for estimation of the vapor pressure. In temperature measurements was used Thermocouple (OMEGA Engineering), Therm 2281-8, Output 0-10 V. 
Acoustic (ultrasound) emissions were taken from a chamber wall with the sensor Ultrasound Sensor Amplifier (Physical acoustic corporation) Model 2/4/6C, Amplification: 20/40/60 dB.

\section{Cavitation number}

Dimensionless number quantifying the cavitation is called cavitation number and is usually denoted as $\sigma$. Cavitation number for nozzles and orifices may be written according classical definition as follows:

$$
\sigma=\left(p_{r}-p_{v}\right) /\left(0,5 v_{r}^{2} \rho\right)
$$

In a case of the orifice is as reference pressure $\mathrm{p}_{\mathrm{r}}$ taken the downstream pressure sufficiently far behind the orifice opening and as a reference velocity $v_{r}$ is taken a velocity in the orifice opening. $p_{v}$ is the vapor pressure of the liquid, $\rho$ is a liquid density.

\section{RESULTS OF EXPERIMENTS}

The goal of the visualization was an estimation of a flow pattern at different values of cavitation number. From the pictures shot by the camera it is possible to estimate a cavitation cloud length which is changing within a certain range.
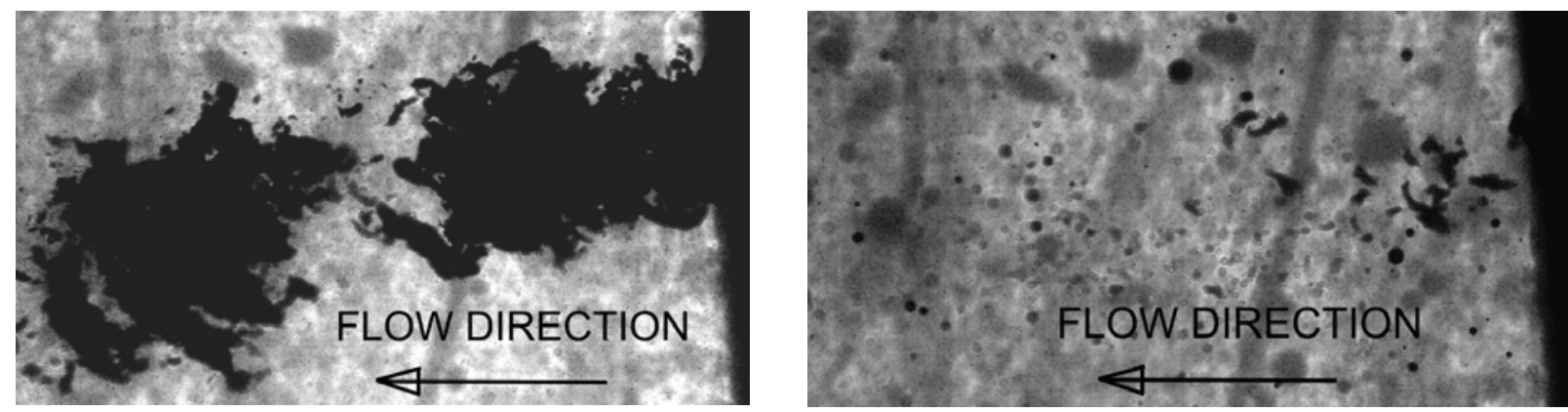

Figure 3: Typical flow patterns of cavitation cloud (left) $\sigma=0,053$ (supercavitation), (right) $\sigma=0,169$.

In the fig. 3 are pictured typical flow patterns for low (left) and high (right) cavitation number. Arrows in the figures denote the flow direction. The cavitation cloud is pictured as dark areas. The pictures are taken at relative high flow speeds - for example a mean flow velocity in micro-orifice in the fig. 3 right is $59 \mathrm{~m} / \mathrm{s}$. In the fig. 4 is evaluated the length of the cavitation cloud (upper and low value) depending on the cavitation number. The range of lengths in the fig. 4 is caused by erratic behavior of the flow. The cavitation cloud length at each cavitation number is changing very rapidly in time. Cavitation intensity decreases with an increased cavitation number and about the value $\sigma=0,175$ supercavitation vanishes. In the orifice itself the cavitation exists also above this value this is shown by experiments in [3], where incipient cavitation was estimated at $\sigma=0,284$ and choked cavitation was estimated at $\sigma=0,242$.

During our experiments were also recorded acoustic (ultrasound) emissions. The ultrasound record was taken from the chamber wall. On the base of acoustic emissions it 
is for example possible to determine leakages of submerged microgaps in hydraulic machines.

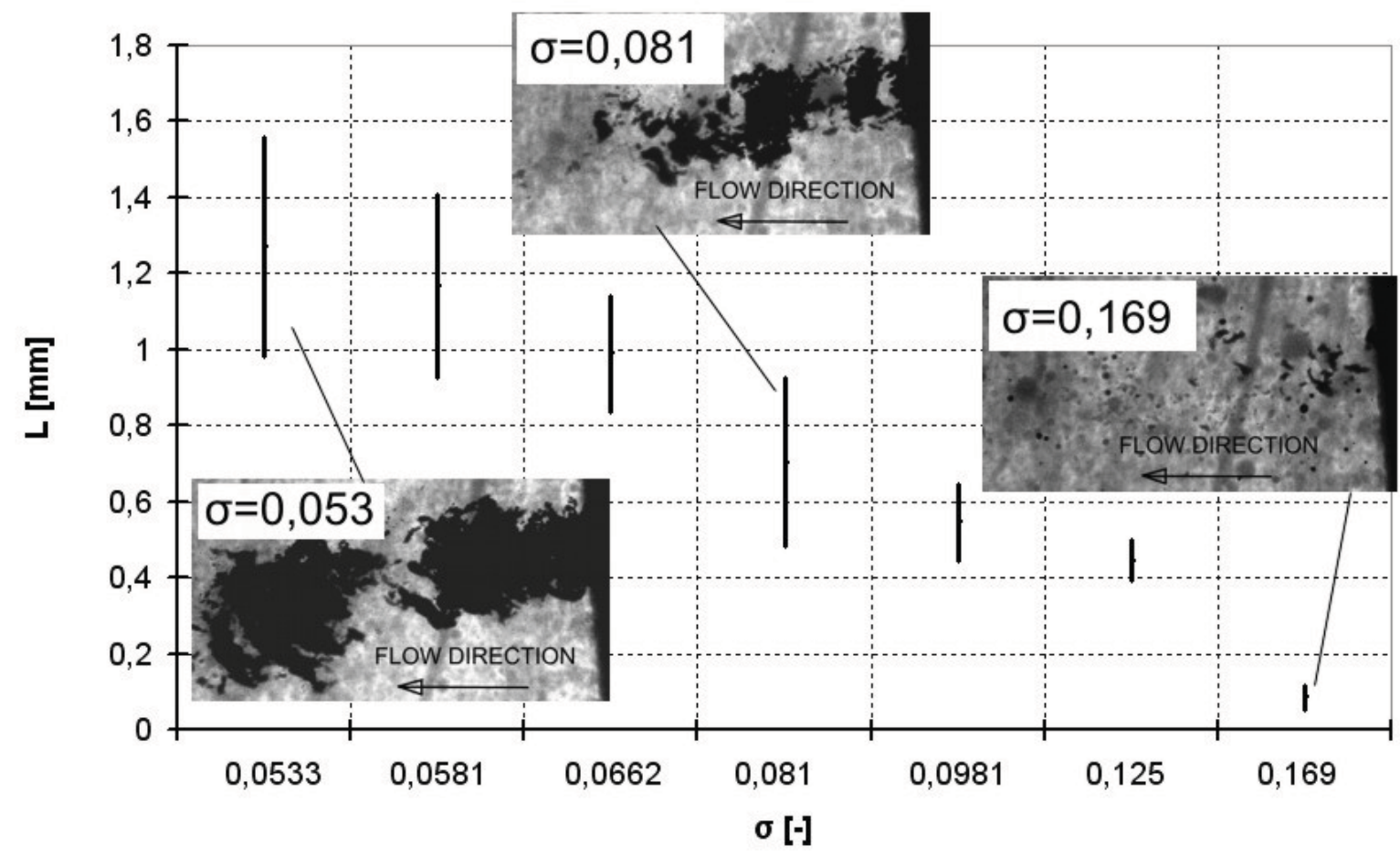

Figure 4: Upper and lower lengths (L) of the cavitation cloud (see fig. 2).
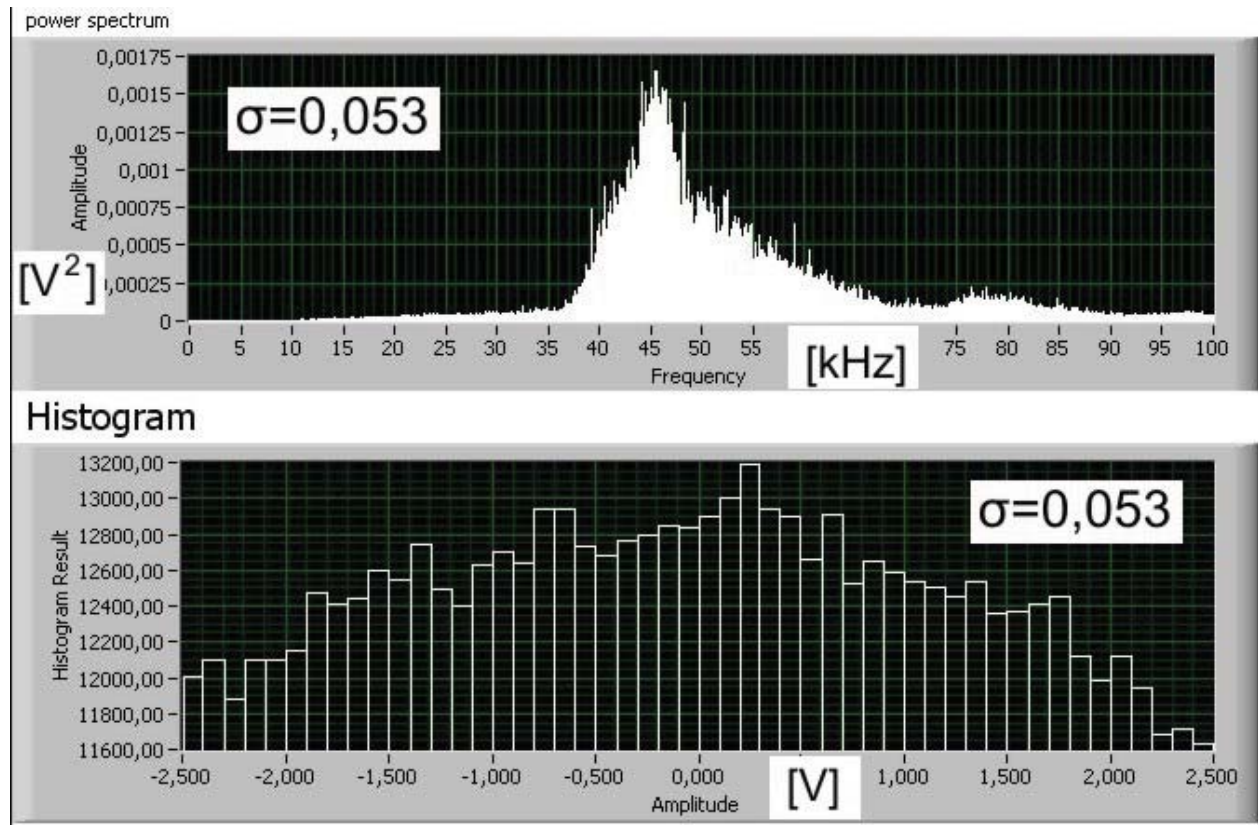

Figure 5: Typical power spectrum and amplitude histogram for supercavitation. 


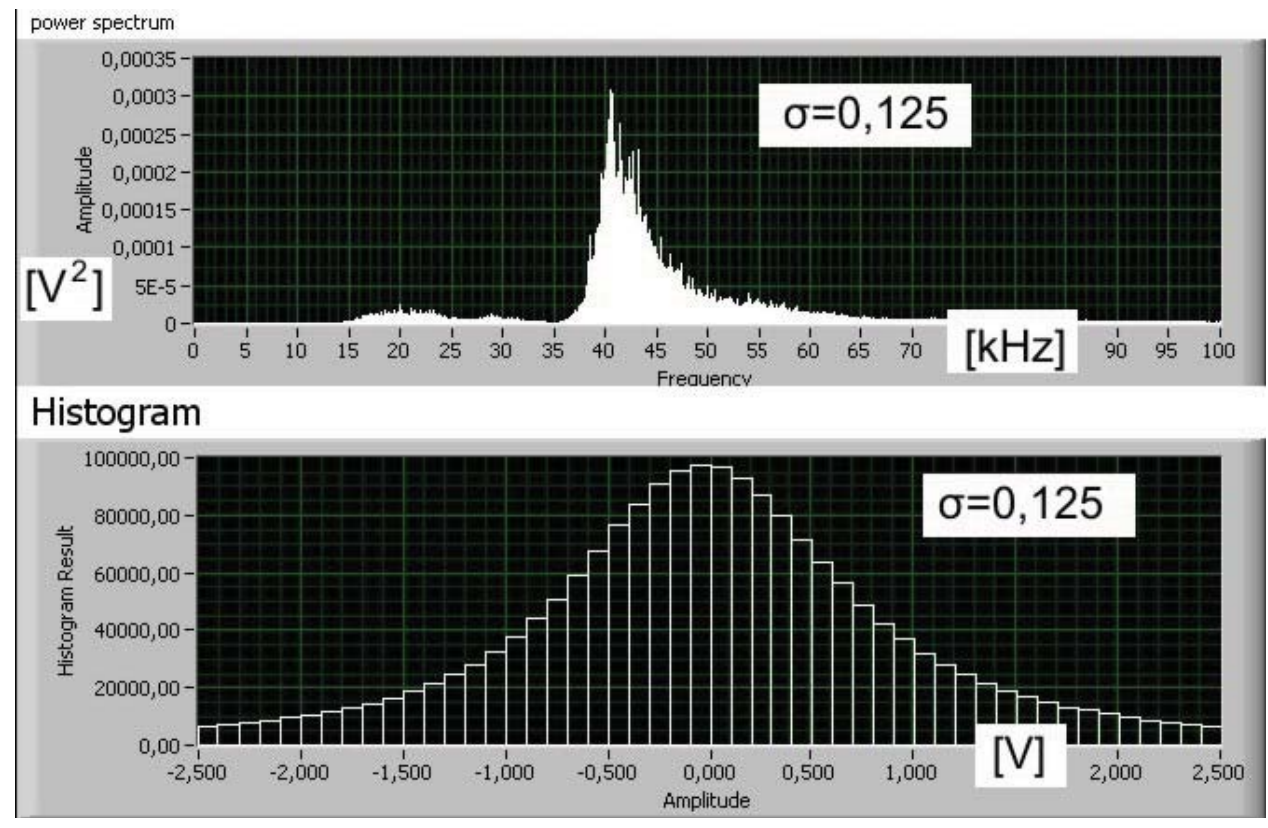

Figure 6: Typical power spectrum and amplitude histogram for cavitation.

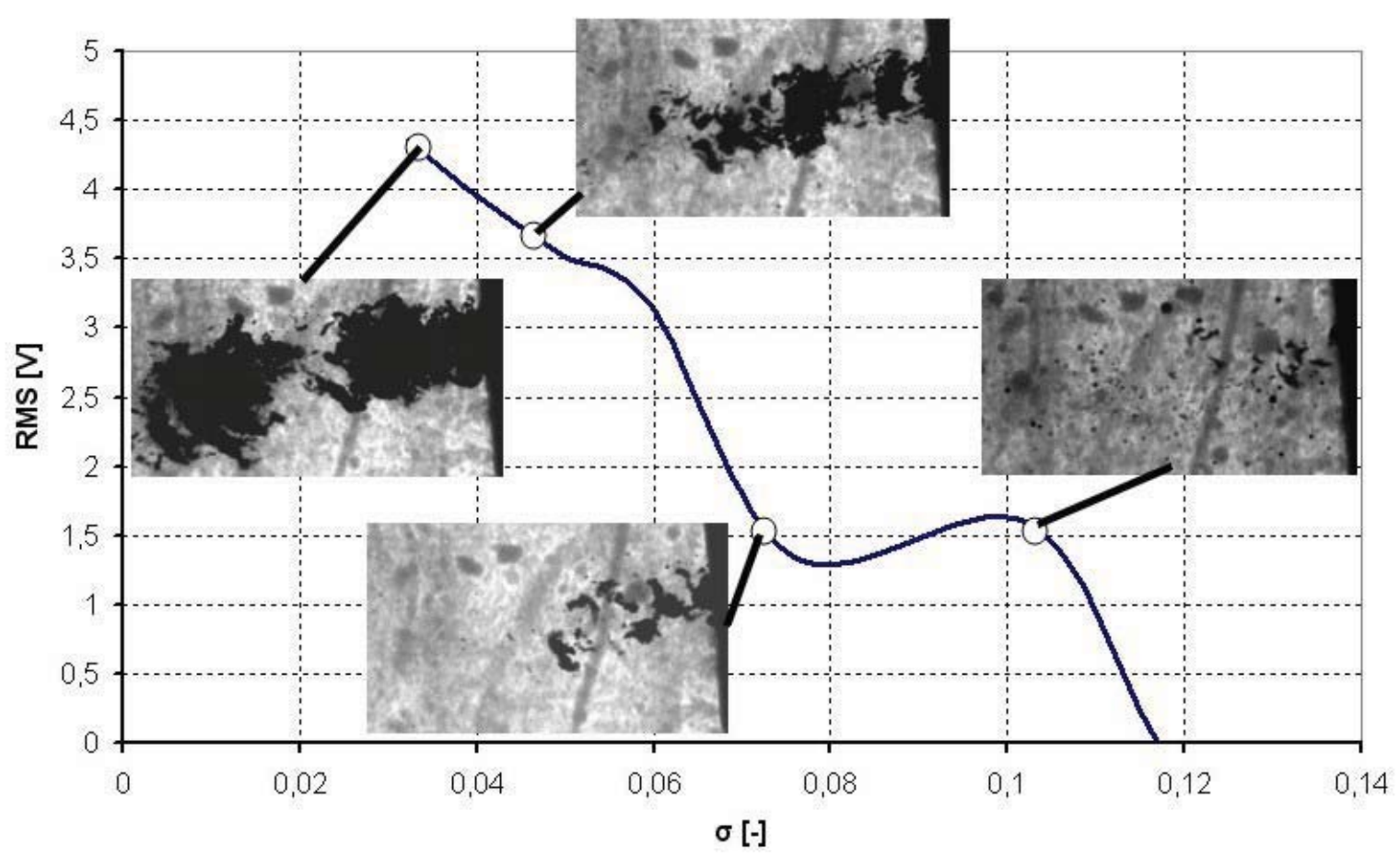

Figure 7: RMS dependence on a cavitation number $\sigma$

In the fig. 5 is a typical power spectrum and an amplitude histogram for the supercavitation (in this case $\sigma=0,053$ ). The frequency range up to $70 \mathrm{kHz}$ corresponds to data from [1]. 
In the fig. 6 is a power spectrum and an amplitude histogram for a cavitation (in this case $\sigma=0,125)$. Cavitation cloud behind the orifice exhibits only a low intensity. But there is a cavitation inside the orifice.

In the fig. 7 is pictured the course of a root mean square (RMS) of the ultrasound record within the range of $\sigma=0,053-0,12$. Interesting is increasing of the RMS value in the point of about $\sigma=0,1$ - shortly before the supercavitation disappearance. This increasing was observed also on other types of orifices.

\section{Conclusions}

Cavitation is a phenomenon depending on the size scale. In this paper is described the test rig designed for the research of cavitation in micro-dimensions. Respect to the fact that the flow velocities are very high, for visualization of flow it was necessary to use the high speed camera. Visualization system using the camera RedLake proved good because obtained images are sufficiently sharp for making of different analysis. It succeeded to measure the length of a cavitation cloud and its dependence on cavitation number. Presented experimental test rig is usable for visualization of a cloud collapse on a solid obstacle.

Besides of visualization was also presented analysis of acoustic emissions (taken from the wall of the chamber) of cavitation phenomenon. Strong dependence of acoustic emissions on a cavitation number was found.

This work was supported by the Scientific Grant Agency VEGA under contract number $1 / 0215 / 11$.

\section{REFERENCES}

[1] Brennen C.E.: Cavitation and Bubble Dynamics, New York, Oxford, Oxford University Press, 1995

[2] Hutli E.A, Nedeljkovic, M. Ilic V.: Visualization of Submerged Cavitating Jet: Part One, 16th Australasian Fluid Mechanics Conference Crown Plaza, Gold Coast, Australia 2-7 December 2007, pages 876-880

[3] Chandan M., Peles Y.: Cavitation in flow through a micro-orifice inside a silicon microchannel, PHYSICS OF FLUIDS 17, 013601 (2005), pages 013601-1, 013601-15 